\title{
Manejo del riesgo en calles Don Pedro y Alfonso, Santo Domingo de Heredia, Costa Rica
}

\author{
Risk management in Don Pedro y Alfonso streets, \\ Santo Domingo de Heredia, Costa Rica
}

\author{
Mario Fernández Arce* \\ Cristian Aguilar Barboza** \\ Oscar Sojo Alemán***
}

Fecha de recibido: 21 de noviembre de 2019

Fecha de aceptado: 10 de septiembre de 2020

\section{Resumen}

El Canal Natural Calle Don Pedro (CNCDP) ha generado erosión lateral e inundaciones. Por lo anterior, ha sido necesario proteger el fondo y las paredes del mismo y transportar más eficientemente sus aguas. Este trabajo resume las obras de mitigación realizadas, lo que incluye la identificación y descripción de las mismas. Estos valiosos aportes a la gestión local de riesgos rara vez son divulgados y valorados por lo que se considera de suma importancia difundirlos. La publicación pretende no solo dar a conocer contribuciones a la gestión de riesgos sino generar datos que alimenten una futura plataforma local de información para gestión de riesgos. La investigación requirió revisión bibliográfica, trabajo de campo y cartografía de las estructuras realizadas. Lo implementado incluye canales de concreto y muros de gaviones.

Palabras clave: canal, erosión, protección, inundación, gaviones, muros.

* Escuela de Geografía, Universidad de Costa Rica, Costa Rica. Correo electrónico: mario.fernandezarce@ucr.ac.cr.

** Escuela de Geografía, Universidad de Costa Rica.

$\star \star \star$ Sociedad civil. 


\section{Abstract}

The Canal Natural Calle Don Pedro (CNCDP) has generated lateral erosion and floods. Therefore, it has been necessary to protect its bottom and walls and transport its waters more efficiently. This work summarizes the mitigation structures built which includes their identification and description. These valuable contributions to local risk management are rarely disclosed and valued for what is considered very important to publish them. The publication aims not only to announce the contributions to risk management but to generate information that feeds a future local information platform for risk management. The research required bibliographic review, fieldwork and mapping of the structures performed. Such structures include channels of concrete and gabion walls.

Key words: channel, erosion, protection, floods, gabions, walls.

\section{Introducción}

Según Fernández et al. (2013), las inundaciones son el segundo peligro más grave en el cantón Santo Domingo de Heredia, Costa Rica. Han causado un importante grado destrucción en los últimos años. En el oficio DSOT-DA-013-10 se muestran los efectos del desbordamiento del canal natural (cual llamaremos Canal Natural Calle Don Pedro - CNCDP) que corre paralelo a las calles Don Pedro (CDP) y Alfonso (CA) (Figura 1), lo que incluye la acumulación de escombros en la urbanización Quizarco.

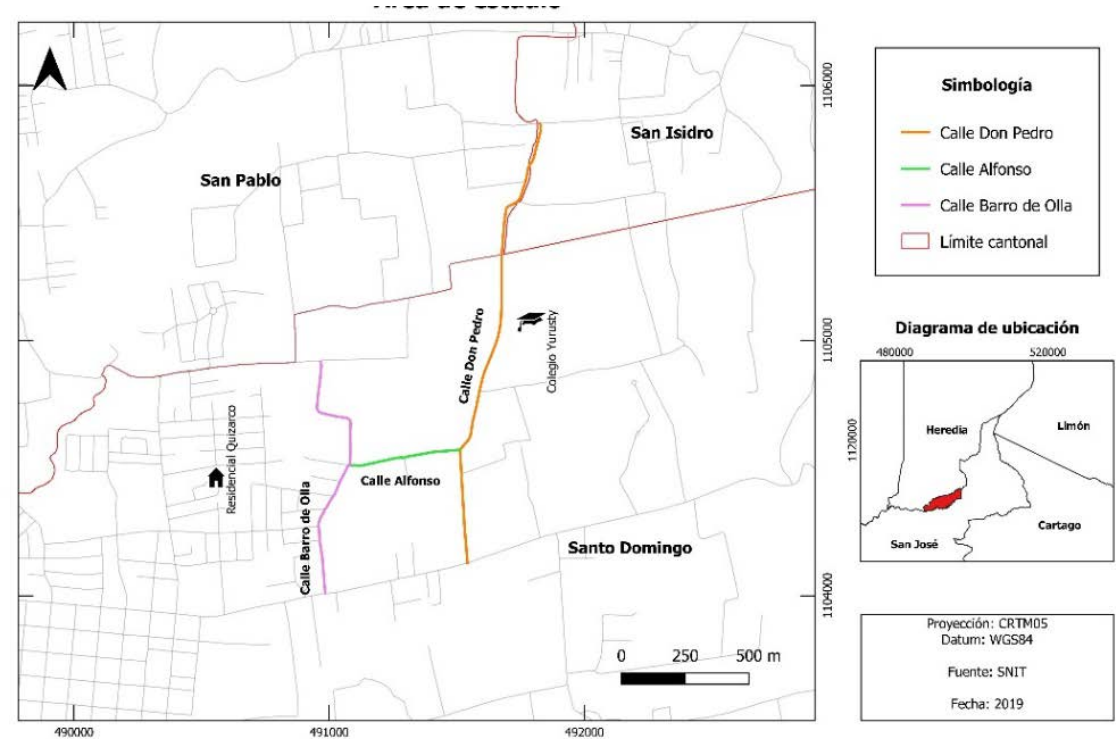

Figura 1. Área de estudio. 
La utilidad práctica del trabajo es informar acerca de lo que está haciendo el Sistema Nacional de Gestión de Riesgos, y en particular el gobierno local de Santo Domingo, para enfrentar la amenaza por desbordamientos e inundación. Esto no solo contribuye a conocer el grado de gestión del riesgo, sino que puede ayudar y estimular a otras entidades a realizar lo mismo u otras medidas de mitigación. Además, los resultados quedan prácticamente listos para ser incorporados en plataformas de información para el manejo de los riesgos.

Para lograr el objetivo, fue necesario en primera instancia, indagar sobre la situación que ha venido generando el CNCDP, lo que implicó revisar las publicaciones hechas al respecto, así como informes y otros documentos escritos. Posteriormente se visitaron las obras para conocerlas, documentarlas y describir sus características. Dichas visitas fueron aprovechadas para determinar las dimensiones de las obras, mediante mediciones simples con una cinta métrica y fotografías.

Los resultados indican que en el territorio de Santo Domingo, prácticamente todo el canal ha sido intervenido a lo largo de las calles Don Pedro y Alfonso. En la primera predominan los muros de gaviones (caja o cesta de forma prismática rectangular, rellena de fragmentos de roca, de mallas metálicas de acero inoxidable o hierro galvanizado con bajo contenido de carbono) hechos para protegerla de la erosión lateral y en la segunda un canal abierto de forma trapezoidal cuyo fin es transportar las aguas sin causar inundaciones.

\section{Metodología}

El primer paso realizado fue una revisión bibliográfica para conocer en detalle las características del CNCDP, los eventos más importantes generados por él y su potencial para generar inundaciones. Esto implicó la búsqueda de artículos científicos, informes técnicos y de situación de la Comisión Nacional de Prevención de Riesgos y Atención de Emergencias (CNE), la Municipalidad de Santo Domingo, el Ministerio de Salud e instituciones de primera respuesta como bomberos, policía y Cruz Roja.

Posteriormente se hizo una serie de visitas al campo. Se aclara que esto incluyó inspecciones hechas años atrás, lo cual resultó muy valioso porque permitió ver la evolución de los trabajos a través del tiempo, lo que indefectiblemente habla de la problemática ocasionada por el canal. Las primeras visitas fueron para simple observación pero conforme se detectó el incremento de las obras, se estimó adecuado cuantificarlas y cartografiarlas. Esto demandó medir las dimensiones de los canales de concreto y de los muros de gaviones, lo cual se hizo con cinta métrica. También se aprovechó para determinar las dimensiones de las obras, algunas de las cuales estaban en plena construcción al momento del trabajo de campo del equipo investigador. 
El último paso fue la cartografía. Se recurrió a la base de datos del Sistema Nacional de Información Territorial (SNIT), y se obtuvo información sobre la extensión del canal, de las calles de interés y de los límites cantonales. En esta fase fue esencial medir la extensión de las estructuras emplazadas y los sitios entre ellas que aún no tienen protección. Cada obra identificada fue georreferenciada en el sistema de proyección CRTM05 y cartografiadas (Figura 2). Tanto los segmentos protegidos como los desprotegidos fueron identificados con puntos (P0-P10) y descritos de la manera más precisa posible.

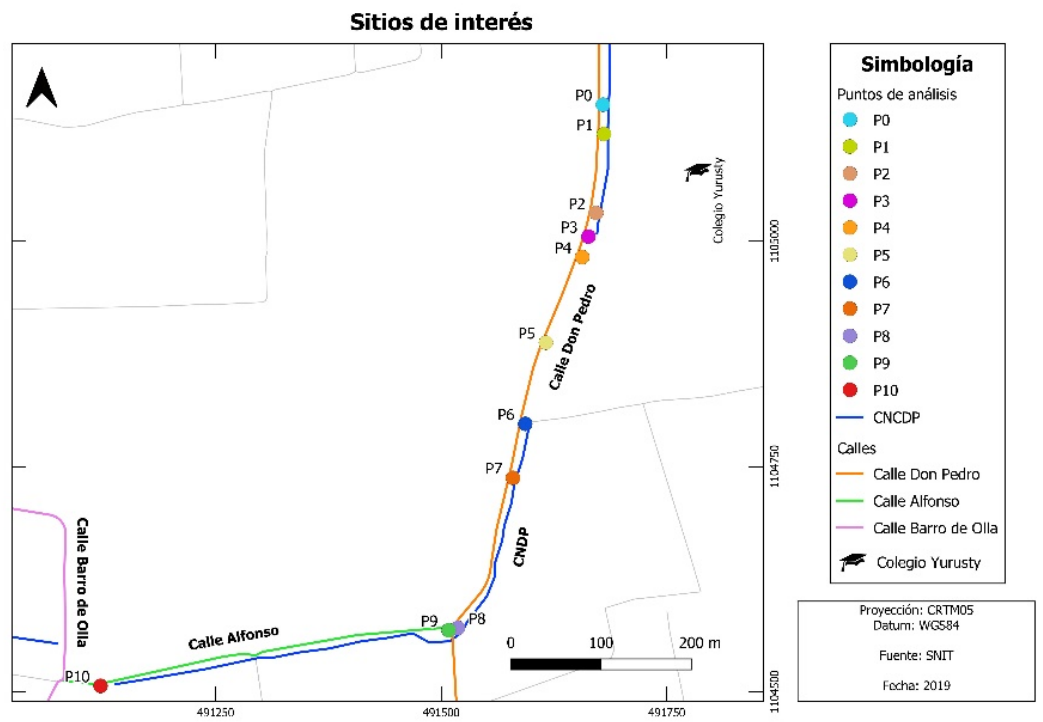

Figura 2. Ubicación de puntos que limitan segmentos del canal, con y sin protección.

\section{Resultados}

La amenaza por inundaciones en el cantón Santo Domingo de Heredia ha sido abordada previamente por Reyes (2012), Fernández et al. (2013), Reyes et al. (2014a) y Reyes et al. (2014b). Como se indica en los anteriores trabajos, el canal natural ha tenido crecidas importantes y repentinas que han dañado una importante vía de comunicación terrestre así como viviendas cercanas. Para evitar estos impactos negativos de los desbordamientos de ríos en general, el Marco de Sendai para la reducción del riesgo de desastres 2015-2030 (instrumento mundial para el manejo de los riesgos) ha establecido como prioridad 3 "Invertir en la reducción del riesgo de desastres para la resiliencia", considerando que las inversiones públicas y privadas para la prevención y reducción del riesgo de desastres, mediante medidas estructurales, son esenciales para aumentar la 
resiliencia económica, social, sanitaria y cultural de las personas, las comunidades, los países y sus bienes, así como del medio ambiente (UNISDR, 2015). La Política y el Plan Nacional de Gestión de Riesgos de Costa Rica tienen como uno de sus ejes la inversión financiera sostenible, infraestructura y servicios (CNE, 2015; CNE, 2016).

En apego a las prioridades del Marco de Sendai y a los ejes de la Política y el Plan Nacional de Gestión de Riesgos de Costa Rica, la Municipalidad de Santo Domingo ha reforzado y consolidado los márgenes inestables del CNCDP mediante acciones localizadas, específicamente, mediante obras de defensa ribereña que tienen como objetivo reducir el impacto de los torrentes e impedir la erosión por la excesiva velocidad del agua. Para ello se ha revestido con concreto el canal natural en unos tramos y se han instalado muros de gaviones en otros. Los revestimientos de concreto en canales abiertos permiten reducir el impacto del flujo de agua sobre el fondo y paredes de los canales; son abiertos porque la parte superior está expuesta a la atmósfera. Los muros de gaviones son obras longitudinales con la misión principal de soportar el empuje activo del terreno. También desvían los flujos de agua y defienden la estabilidad de los márgenes de quebradas y ríos sujetos a erosión. El agua puede pasar por ellos pero no debe acumularse en su parte trasera porque ello aumentaría las fuerzas de empuje.

\section{Obras en calle Don Pedro (CDP)}

Para proteger esta calle de la erosión lateral causada por torrentes de agua se ha construido un conducto cerrado frente al Colegio Yurusty (Reyes, 2012; Fernández et al., 2020 en revisión) y muros de gaviones. El primero consistió en un revestimiento del desagüe con block y concreto. Los gaviones son elementos de forma prismática rectangular, fabricados con malla hexagonal hecha con alambre resistente, rellenos de fragmentos de roca que se colocan frente a suelos inestables para evitar deslizamientos o erosión. Son de fácil instalación y muy adecuados porque se integran armoniosamente al entorno, son permeables y no desarrollan presiones hidrostáticas.

En calle Don Pedro, se usaron gaviones tipo caja confeccionados con malla hexagonal de doble torsión, tipo 8x10 (ASTM A 975-97) a partir de alambre de acero (bajo contenido de carbono), galvanizado, con diámetro $2.40 \mathrm{~mm}+\mathrm{PVC}$. El recubrimiento de PVC es de $0.4 \mathrm{~mm}$ de espesor, color gris, para alcanzar un diámetro total de $3.2 \mathrm{~mm}$. Los diafragmas o paños divisorios se colocaron a un metro de distancia entre uno y otro. Para realizar amarres y elaborar tensoras se utilizó alambre adicional con las mismas características de la malla, diámetro de $2.20 \mathrm{~mm}+\mathrm{PVC}$. La cantidad del mismo será de $8 \%$ sobre el peso de los gaviones de un metro de la altura y de $6 \%$ para los de $0.50 \mathrm{~m}$ de altura. 
También se usaron gaviones tipo colchón de Reno PVC confeccionados en malla hexagonal de doble torsión, tipo 6x8 (ASTM 975-97), a partir de alambre de acero (bajo contenido de carbono), galvanizado + PVC, con diámetro $2.00 \mathrm{~mm}$, de espesor mínimo de $0.40 \mathrm{~mm}$ (ASTM 975-97). Los colchones reno deben ser de diafragmas de pared doble, moldeados de metro en metro durante el proceso de fabricación a partir del paño base, formando un único elemento. El alambre del mismo tipo, para las operaciones de amarre y atirantado, con diámetro de $2.2 \mathrm{~mm}$ y en la proporción de $5 \%$ sobre su peso.

En lo que concierne a colocación y montaje, primero se desdobla el material sobre una superficie rígida y se levantan los laterales y los diafragmas para formar una caja. Luego se juntan los cantos superiores de los paneles con los alambres gruesos que salen de la red. Se fija el alambre de amarre en el canto inferior de las aristas y se amarran alternando vueltas simples y dobles en cada malla. Es preciso sujetar varias cajas en grupos, llevarlas junto a los ya colocados y amarrarlas a lo largo de todas las aristas en contacto. Antes del llenado, hay que tensar después de colocar y amarrar varios gaviones. El llenado se hace en tres etapas: 1) Primero hasta un tercio de la capacidad total, 2) colocar los tirantes y llenar hasta dos tercios de la capacidad total y, 3) colocar nuevamente los tirantes y finalizar el llenado hasta $305 \mathrm{~cm}$, por arriba de la altura del gavión. No llenar una caja sin que la caja de al lado este parcialmente llena. Finalmente, se doblan las tapas y se amarran con el mismo tipo de costura. El trabajo deberá realizarse de forma continua y de conformidad con la metodología de trabajo.

Los fragmentos de rocas empleados son en su mayoría de origen volcánico, principalmente andesíticos. Son de roca quebrada o de canto rodado, de buena calidad. Su tamaño varía de 10 a $30 \mathrm{~cm}$ con un porcentaje de fragmentos de mayor a menor tamaño máximo del $20 \%$. Los trozos de menor diámetro pueden utilizarse para rellenar los espacios vacíos entre clastos más grandes.

En algunos sitios se requirió realizar rellenos laterales, en los cuales se solicitó las siguiente especificaciones: 1) Compactación en capas de máximo $25 \mathrm{~cm}$ y que alcanzara el 95\% del proctor modificado; 2) el tamaño mínimo de los fragmentos del material de relleno debió ser por lo menos $30 \mathrm{~mm}$ mayor que las aberturas de la malla de la canasta y el tamaño máximo de $250 \mathrm{~mm}$; 3) el desgaste del material al ser sometido a ensayo en la máquina de los Ángeles, según norma AASTHO T-96, debió ser inferior al 50\%; 4) Su capacidad de absorción de agua debió ser inferior al $2 \%$ por peso, para su determinación de la gravedad específica se fragmentó una muestra representativa de las rocas y se ensayó de acuerdo con la norma AASTHO T-85.

A continuación se describen los segmentos protegidos y desprotegidos del CNCDP en la zona de estudio. 
- Segmento $P_{0}-P_{1}$. Este tramo del CNCDP está revestido con concreto, obra que se denomina Canal Cerrado (CC). En abril del 2012 el CC estaba en plena construcción y se pudo ver que el fondo fue revestido de concreto y sus paredes fueron reforzadas con una hilera de block de $2.4 \mathrm{~m}$ de altura con respecto al lecho de la quebrada.

La obra tiene una longitud de $113.6 \mathrm{~m}$; inicia al norte de la entrada al Colegio Yurusty y termina al sur de la misma. El ancho de esta es $1.9 \mathrm{~m}$ y un pequeño muro de $60 \mathrm{~cm}$ evita que el suelo lateral se erosiones y se desplome sobre el techo del canal.

Es probable que la inestabilidad de la calle provocada por los veloces flujos de agua afectara de manera indirecta a la población del colegio, razón por la cual se requería una solución al problema. Se le colocó techo quizá por protección, probablemente para evitar que alguien pueda caer en el canal, lo cual podría resultar mortal. Un aspecto a destacar de esta obra es que redujo la fricción del agua con el suelo por lo que la misma puede fluir con mayor velocidad, lo cual puede influir en la erosión de las paredes laterales en otros sitios sin protección. Un comentario adicional es que el gran espacio que ha quedado frente a la obra es usado como estacionamiento eventualmente.

- Segmento $P_{1}-P_{2}$. Después del CC, entre los puntos $P_{1}$ y $P_{2}$, hay un corto tramo de $15.20 \mathrm{~m}$ sin protección. No se conoce la razón por la que ese sector está desprotegido pero se asume que probablemente la erosión ha sido insignificante allí quizá porque el canal natural es recto.

- Segmento $P_{2}-P_{3}$. A continuación, un segmento de $8 \mathrm{~m}\left(\mathrm{P}_{2}-\mathrm{P}_{3}\right)$ en el que la ribera del canal está protegido con un muro de concreto y un gavión. Este último es de un metro de ancho y fue hecho para proteger la base de un poste del tendido eléctrico.

- Segmento $P_{3}-P_{4}$. El segmento $P_{3}-P_{4}$ tiene 24 metros y no está reforzado por lo que no cuenta con protección.

- Segmento $\mathrm{P}_{4}-\mathrm{P}_{5}$. Este tramo del canal es de $107.4 \mathrm{~m}$ y comienza con un puente de concreto de $7.3 \mathrm{~m}$ a partir del cual inicia un muro de gavión que se extiende hasta $P_{5}$. La pared vertical es de 1 metro de ancho y 1.85 metros de altura con respecto al lecho. Es una estructura rectangular hecha con malla de alambre rellenada con fragmento de roca.

- Segmento $P_{5}-P_{6}$. El siguiente sector, el que se extiende de $P_{5}-P_{6}$, es de $101.3 \mathrm{~m}$ y corresponde con una franja de viviendas que fueron construidas a la par del y frente al canal. Por tanto, todo ese tramo está cubierto por estructuras de concreto que comunican las casas con la calle. Se desconoce si en este segmento se reforzaron las paredes del canal natural antes de hacer las obras de concreto pero es probable que si porque tales obras aparentan ser pesadas. 
- Segmento $P_{6}-P_{7}$. Este sector es de $74.8 \mathrm{~m}$ y no cuenta con protección. En él hay pocas evidencias de erosión y está reforzado de manera natural con vegetación muy tupida, básicamente pasto y algunas plantas de mediano tamaño.

- Segmento $P_{7}-P_{8}$. En $P_{7}$ comienza un segmento de gaviones construidos en diferentes momentos, siendo el más reciente del 2019. Al igual que el tramo anterior, es una pared vertical de malla rellena con fragmentos de roca y malla con canasta en el fondo (Figura 3). Las dimensiones de la pared son $150 \mathrm{~m}$ de largo, un metro de ancho y $1.9 \mathrm{~m}$ la altura de la pared vertical. El ancho de la malla basal es de $2.10 \mathrm{~m}$. El eje longitudinal de los clastos varía entre 10 a $12 \mathrm{~cm}$ pero en otros sectores el rango fluctúa entre 16 a $22 \mathrm{~cm}$. La serie termina en $\mathrm{P}_{8}$ donde hay un para de alcantarillas que permiten el paso del agua por debajo de la calle Don Pedro (sector $\mathrm{P}_{8} \cdot \mathrm{P}_{9}$ ) y la desvían hacia calle Alfonso.

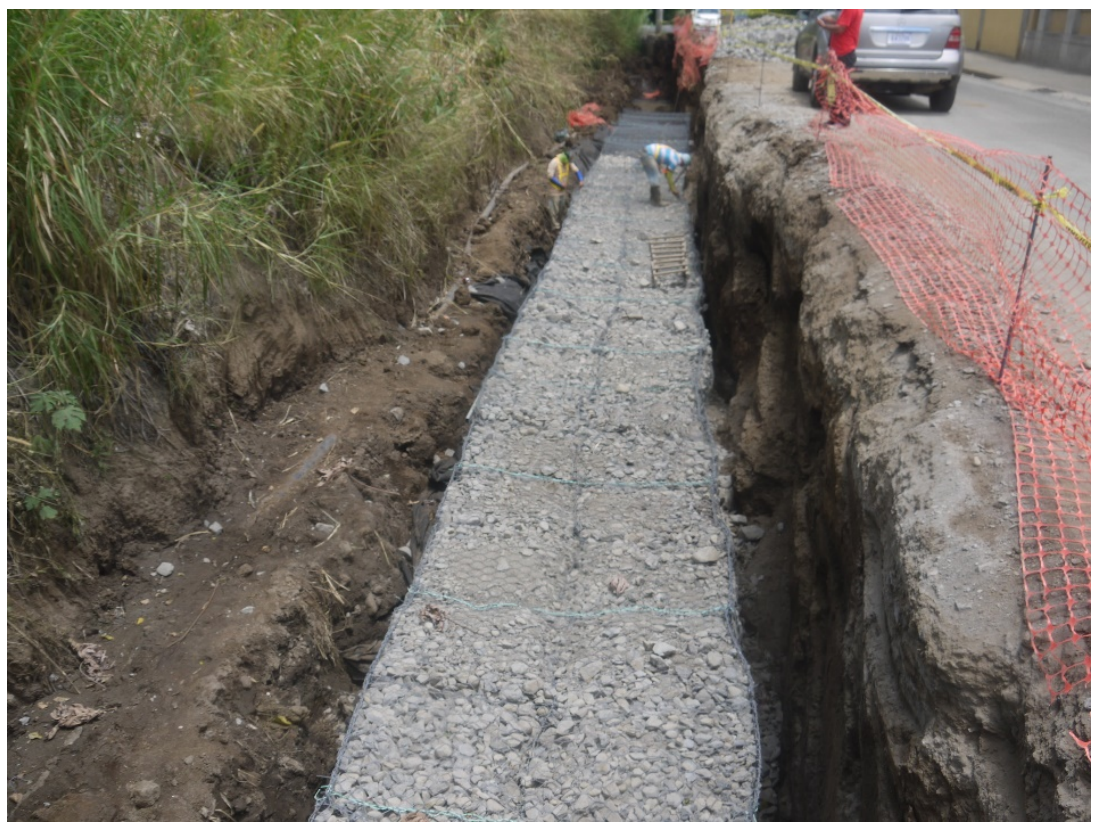

Figura 3. Construcción del último gavión en 2019. La foto fue tomada el 1 de mayo de 2019 cuando la construcción estaba en pleno apogeo. La vista es hacia el sur.

\section{El Canal Abierto de Calle Alfonso (CA)}

La calle Alfonso comunica a calle Don Pedro con la calle Barro de Olla (CBO), la cual limita con la urbanización Quizarco. CA se inundaba por completo cuando el 
torrente de agua que bajaba por el canal natural adyacente a CDP era considerable y extraordinario.

Reyes et al. (2014) indican que esta calle es afectada por pequeñas inundaciones que se deben a desbordamiento del agua del canal por falta de capacidad del mismo. Para subsanar esa anómala situación, se hizo un canal abierto de forma trapezoidal, en el que el agua fluye por la acción de la gravedad, rodeada por un contorno sólido. Se construyó en concreto de $210 \mathrm{~kg} / \mathrm{cm}^{2}$ (con batidora), con malla electrosoldada no. 2, sobre una cama de concreto de pobre resistencia $105 \mathrm{~kg} / \mathrm{cm}^{2}$ de $10 \mathrm{~cm}$ de espesor. El espesor del canal es de $15 \mathrm{~cm}$ y sus dimensiones son variables, con una altura promedio de $1.4 \mathrm{~m}$, base promedio de $1.2 \mathrm{~m}$, para un área de caudal de entre $1.7 \mathrm{~m}^{2}-2 \mathrm{~m}^{2}$. No se utilizó ningún aditivo.

Se realizó por etapas, la última de las cuales se ejecutó en el primer semestre del 2012. El inicio de la obra se ubica cerca de la intersección entre CDP y CA $\left(P_{9}\right)$ y el fin cerca del cruce entre CA y CBO, que corresponden con el P10. En esencia, la obra consiste en el revestimiento del fondo y paredes del canal natural con concreto. Aunque las dimensiones se reducen, hay dos orificios que favorecen el paso del agua y evitan la acumulación de la misma al pasar a conductos cerrados.

La obra es robusta por lo que ha logrado evitar la inundación de la calle. Quizá sea recomendable colocar rejas en su parte superior porque el canal es muy ancho y si una persona cae dentro de él en el momento de un gran torrente podría perecer ahogado, máxime que no cuenta con rugosidades o algún otro detalle que le permita a la víctima sostenerse.

Tanto para los gaviones como para el canal trapezoidal se realiza mantenimiento anual antes de invierno, lo que implica normalmente una limpieza manual y mecanizada de todo el canal y hacer auscultación visual de algún posible daño o deterioro para ver si es necesario ejecutar alguna mejora.

Las obras realizadas han logrado contener la erosión lateral en la calle Don Pedro y con ello, ha sido posible mantener la comunicación terrestre entre los cantones Santo Domingo y San Isidro. Esto es relevante porque el alto tráfico vehicular de hoy día requiere muchas rutas activas y en buen estado. El canal trapezoidal de concreto permite que el agua discurra por el canal natural sin fricción, evitando la formación de embalses, los desbordamientos que inundaban la calle Alfonso y los daños a viviendas cercanas al CNCDP. La construcción de dichas obras ha confirmado las estimaciones del Marco de Sendai acerca de su utilidad para reducir el riesgo y aumentar la resiliencia.

El levantamiento y descripción de las obras antes mencionadas serán parte de la plataforma de información para el manejo de los riesgos del cantón, de la cual ya hay una versión que debe ser mejorada primero y luego implementada. Este artículo podrá quedar insertado a dicha plataforma mediante un vínculo. 


\section{Conclusiones}

Las obras de mitigación de tipo estructural construidas para evitar la erosión lateral del canal natural a lo largo de la calle Don Pedro incluyen un canal de concreto cerrado y muros de gaviones de caja y de colchón. La longitud del primero es $113.6 \mathrm{~m}$ y la sumatoria de la longitud de los gaviones es $250 \mathrm{~m}$ aproximadamente. Estos últimos son mallas hexagonales de doble torsión, hechas con alambre de acero galvanizado, con diámetro $240 \mathrm{~mm}+\mathrm{PVC}$. Dichas mallas fueron llenadas con fragmentos de roca quebrada o de cantos rodado, de tamaño variable entre 10 y $30 \mathrm{~cm}$.

En la calle Alfonso se hizo un canal trapezoidal de concreto, con malla electrosoldada no.2, sobre una cama de concreto de $10 \mathrm{~cm}$ de espesor. Sus dimensiones son variables, con una altura promedio de $1.4 \mathrm{~m}$, base promedio de $1.2 \mathrm{~m}$, para un área de caudal de entre $1.7 \mathrm{~m}^{2}-2 \mathrm{~m}^{2}$. Esta estructura es de 600 metros de longitud aproximadamente.

El impacto del pequeño canal natural paralelo a las calles Don Pedro y Alfonso, que incluye importantes inundaciones y severos daños a dichas calles, ha sido contrarrestado con las obras antes mencionadas. Con ellas, la Municipalidad de Santo Domingo de Heredia ha demostrado que está realizando gestión de riesgo en su territorio y ha logrado reducir el impacto de los torrentes de agua que fluyen por el canal natural. Además, lo hecho se apega a las prioridades del Marco de Sendai y tanto a la Política como al Plan Nacional de Gestión del Riesgo de Costa Rica.

\section{Referencias}

Comisión Nacional de Prevención de Riesgos y Atención de Emergencias de Costa Rica (CNE) (2015). Política Nacional de Gestión del Riesgo 2016-2030 (60pp.). San José, Costa Rica.

Comisión Nacional de Prevención de Riesgos y Atención de Emergencias de Costa Rica (CNE) (2016). Plan Nacional de Gestión del Riesgo 2016-2030 (60 pp.). San José, Costa Rica.

Dirección de Servicios y Ordenamiento Territorial (DSOT), Municipalidad de Santo Domingo (2010). Informe general de la Emergencia del martes 20 de julio de 2010, acontecido en el cantón de Santo Domingo. Informe interno, DSOT-DA-013-10, (14 pp). Costa Rica.

Fernández, M.; Borges, J.; Meléndez, G.; Mora, F.; Mora, J. \& Muñoz, Ch. (2013). Análisis de Gestión de Riesgo de Inundación en la ciudad de Santo Domingo de Heredia. En Adamson, M., Castillo, F., Costa Rica en el tercer milenio: desafíos y propuestas para la reducción de vulnerabilidad ante los desastres.

Fernández, M.; Muñoz, R., Sojo, O. (2019). Obras de mitigación en Santo Domingo de Heredia. En torno a la prevención (sometido).

Reyes, J. (2012). Determinación del Riesgo de Desastres en el cantón Santo Domingo de Heredia mediante sistemas de información geográfica. Tesis de Maestría, Universidad de Costa Rica. 
Reyes, J.; Fernández, M.; Grinesky, S. \& Collins, T. (2014) Natural Hazards in Santo Domingo de Heredia, Costa Rica, Central América. Natural Science 6(3): 121-129.

Reyes, J., Fernández; M., Grinesky, S. \& Collins, T. (2014). Spatial Analysis of Disaster Risk in Santo Domingo de Heredia, Costa Rica, Central America. Journal of Geography and Geology 6(3): 123-13. DOI: https://www.doi.org/10.5539/jgg.v6n3p123

UNISDR, (2015). Marco de Sendai para la Reducción del Riesgo de Desastres 2015-2030, 40 pp. 